\title{
Proof of a conjecture of McKay
}

by

Krister Segerberg (Åbo Akademi, Finland)

Abstract. We show how to construct for each finite intuitionistic tree model a finite binary intuitionistic tree model equivalent to the first one if only disjunction free formulas are considered. This confirms McKay's conjecture, viz., that the ICN -fragment of Heyting's propositional logic is determined by the class of finite binary trees.

Let $T$ be the class of intuitionistic Kripke structures $\langle U, \leqslant\rangle$ that take the form of a finite tree, and let $T_{2}$ be the subclass of $T$ whose members are the binary trees. It is well-known that the formulas valid in $T$ are exactly those that are intuitionistically valid (see [2]). It is also known that there are formulas that are valid in $T_{2}$ but not in $T$ (see [1]). o. G. McKay has conjectured (private communication) that as far as ION-formulas go exactly the same are valid in $T$ and $T_{2}$. (ION-formulas, or disjunction free formulas, are built from propositional letters nsing at most implication, conjunction, and negation - disjunction is not allowed.) In this note we shall prove the correctness of his conjecture:

TEEOREM. Every ION-formula rejected by a finite tree model is also rejected by some finite binary tree model.

We shall describe a construction that transforms certain tree models into binary tree models. Suppose $U=\langle U, \leqslant, \nabla\rangle$ is a finite tree model in which each node has at least two immediate descendants or none (1). Suppose that $U$ has $p$ elements, and let $\left\langle u_{0}, \ldots, u_{p-1}\right\rangle$ be an arbitrary entmeration of the elements of $U$. We let $\mho_{0}=\mho$ and define $\left\langle u_{0}, \ldots, u_{p-1}\right\rangle$ as the standard entumeration of $\mathcal{U}_{0}$. Now suppose that $\mathcal{U}_{n}=\left\langle U_{n}, \leqslant, \nabla_{n}\right\rangle$ and the standard enumeration $\left\langle u_{0}, \ldots, u_{p+n-1}\right\rangle$ of $\mathcal{W}_{n}$ have been defined. If: $u_{n}$ is not a binary tree, then some element must have more than two immerliatie descendants; let $x$ be the first one in the standard enumeration of $\mathcal{U}_{n}$ that has, and let $y_{0}$ and $y_{1}$ be the first two elements in that same enumeration that are immediate descendants of $x$. Pick any object $z \notin U_{n}$. Wo define:

$$
u_{p+n}=z, \quad U_{n+1}=U \cup\{z\} .
$$

(1) As nsual, if $\langle U, \leqslant\rangle$ is a Kripke structure, then $v$ is a descendant of $u$ if $u \leqslant v$ and $u \neq v$; if in addition for all $w, u \leqslant w \leqslant v$ only if $u=w$ or $v=w$, then $v$ is an immediate descendant of $u$. 
For $u \in U_{n}$, define

$$
\begin{array}{lll}
u \leqslant z & \text { iff } & u \leqslant x \text { or } u=z, \\
z \leqslant u & \text { iff } & u=z \text { or } y_{0} \leqslant u \text { or } y_{1} \leqslant u .
\end{array}
$$

For every propositional letter $p$, let

$$
V_{n+1}(\boldsymbol{p})= \begin{cases}V_{n}(\boldsymbol{p}) \cup\{\boldsymbol{z}\}, & \text { if } y_{0}, y_{1} \in \nabla_{n}(\boldsymbol{p}) \\ V_{n}(\boldsymbol{p}), & \text { otherwise }\end{cases}
$$

Then put $\mathcal{U}_{n+1}=\left\langle U_{n+1}, \leqslant, V_{n+1}\right\rangle$, and define $\left\langle u_{0}, \ldots, u_{p+n-1}, u_{p+n}\right\rangle$ as the standard enumeration of $\mathcal{U}_{n+1}$.

Since $U$ is finite this procedure will terminate after a finite number of steps, say $t$. (Actually, $t \leqslant p-3$.) Note that $\mathcal{U}_{t}$ will be a finite binary tree. Then:

LEMina. Suppose $\alpha$ is an ION-formula and $x \in U_{n}$, for some $n>0$.

(i) If $x \in U_{n-1}$, then $\mho_{n} \vDash_{x} \alpha$ if and only if $\varkappa_{n-1} \vDash_{x} \alpha$.

(ii) If $x \notin U_{n-1}$, then $\mathcal{U}_{n} \vDash_{x} \alpha$ if and only if, for every immediate descendant $y$ of $x$ in $\mathcal{U}_{n}, \mathcal{U}_{n} \vDash_{y} \alpha$.

Proof. It should be enough to give the following parts of the full inductive proof. Assume that the lemma holds for $n$ and two formulas $\beta$ and $\gamma$. We will show that the lemma holds for $n$ and the more complex formulas $\beta \wedge \gamma$ and $\beta \rightarrow \gamma$.

The case of $\wedge$. If $x \in V_{n-1}$, then $\mathcal{U}_{n} k_{x} \beta \wedge \gamma$ iff $\mathcal{u}_{n} k_{x} \beta$ and $\mathcal{U}_{n} k_{x} \gamma$ iff, by the induction hypothesis, $\mathcal{U}_{n-1} k_{x} \beta$ and $\mathcal{U}_{n-1} k_{x} \gamma$ iff $\mathcal{u}_{n-1} k_{x} \beta \wedge \gamma$.

Assume instead that $x \notin U_{n-1}$. Then there are exactly two immediate descendants of $x$, say $y_{0}$ and $y_{1}$. If $\mathcal{U}_{n} \vDash_{x} \beta \wedge \gamma$ then by heredity $\mathcal{U}_{n} \vDash_{y_{0}} \beta \wedge \gamma$ and $\mathcal{U}_{n} \vDash_{y_{1}} \beta \wedge \gamma$. If $\mathcal{U}_{n} \neq_{x} \beta \wedge \gamma$ then either $\mathcal{U}_{n} \not \neq_{x} \beta$ or $\mathcal{U}_{n} \not \neq_{x} \gamma$, so by the induction hypothesis either $\mathcal{U}_{n} \mid \neq y_{0} \beta$ or $\mathcal{U}_{n} \mid \neq_{y_{1}} \beta$ or $\mathfrak{W}_{n} \mid \neq y_{y_{0}} \gamma$ or $\mathcal{u}_{n} \mid \neq y_{1} \gamma$, and therefore either $\mathcal{U}_{n} \mid \neq_{\nu_{0}} \beta \wedge \gamma$ or $\left.\mathcal{u}_{n}\right|_{y_{1}} \beta \wedge \gamma$.

The case of $\rightarrow$. First assume that

$$
\mathcal{U}_{n} F_{x} \beta \rightarrow \gamma \text {. }
$$

If $x \in \nabla_{n-1}$, then note that for every descendant $y$ of $x$ in $\mathcal{W}_{n}, \mathcal{U}_{n} F_{y} \beta$ only if $\mathcal{U}_{n} k_{y} \gamma$, and that every descendant of $x$ in $\mathcal{U}_{n-1}$ is a doscendant of $x$ in $\mathcal{U}_{n}$; hence an application of the induction hypothesis yields $\mathcal{u}_{n-1} k_{x} \beta \rightarrow \gamma$. If $x \notin U_{n-1}$, then observe that by heredity $\mathcal{U}_{n} F_{z} \beta \rightarrow \gamma$ for every descendant $z$ of $x$, and a fortiori for the immediatio ones.

Next assume that

$$
\mathcal{u}_{n} \mid \neq x \beta \rightarrow \gamma
$$

It follows that there is some descendant $y$ of $x$ in $\mathcal{W}_{n}$ such that

$$
\begin{aligned}
& \cup_{n} F_{y} \beta, \\
& \cup_{n} \mid \neq_{y} \gamma .
\end{aligned}
$$

Cas e I. $x \in U_{n-1}$. We assume, with no loss of generality, that $y \in U_{n-1}$. (For suppose that $y \notin U_{n-1}$. Then, by (2) and the induction hypothesis, there would be some immediate descendant $z$ of $y$ in $\mathcal{U}_{n}$ such that $\mathcal{U}_{n} \mid \vDash_{z} \gamma$. From (1) it follows by heredity that $\mathcal{U}_{n} \vDash_{z} \beta$. Moreover $x \leqslant z$ and, since $z \neq y, z \in U_{n-1}$.) From (1) and (2), by the induction hypothesis,

Hence

$$
u_{n-1} \vDash_{y} \beta, \quad \mathfrak{U}_{n-1} \neq_{y} \gamma
$$

$$
u_{n-1} \mid \neq x \beta \rightarrow \gamma \text {. }
$$

Case II. $x \notin U_{n-1}$. Let $u_{0}$ and $u_{1}$ be the two immediate descendants of $x$ in $\mathcal{u}_{n}$. If $x \neq y$ then let $z$ be the element in $\left\{u_{0}, u_{1}\right\}$ such that $z \leqslant y$. By (1) and (2),

Hence by heredity

$$
u_{n} \mid \neq{ }_{\nu} \beta \rightarrow \gamma
$$

$$
\mathrm{w}_{n} \mid \neq_{z} \beta \rightarrow \gamma \text {. }
$$

On the other hand, if $x=y$ then (1) and (2) yield

$$
u_{n} k_{x} \beta, \quad u_{n} \neq x \gamma \text {. }
$$

Hence by the induction hypothesis both $\mathcal{U}_{n} k_{u_{0}} \beta$ and $\mathcal{U}_{n} k_{u_{1}} \beta$, and either $\mathcal{u}_{n} \mid \neq u_{0} \gamma$ or $\mathcal{u}_{n} \mid \neq u_{u_{1}} \gamma$. Consequently either $\mathcal{u}_{n} \mid \neq_{u_{0}} \beta \rightarrow \gamma$ or $\mathcal{u}_{n} \mid \neq{ }_{u_{1}} \beta \rightarrow \gamma$.

Coroularx. For every $x \in U_{0}$ and every ION-formula $\alpha$,

$$
\mathcal{U}_{0} k_{x} \alpha \text { if and only if } \mathcal{น}_{t} k_{x} \alpha \text {. }
$$

Proof. Follows by $t$ applications of condition (i).

The encllary establishes the theorem, for if $U$ is a finite tree model rejecting any formula $\alpha$ and there are nodes of $น$ each having exactly ono immodiatio doscendant, then it is easy to embed $\mathcal{U}$ in a finite tree model $u^{*}$ which also rojets $\alpha$ and in which each node has at least two immodiato rlescendants or none. When our construction is applied to $น^{*}$ a finite binary tree model results in which $\alpha$, if ION, will fail.

Finally, leti ns pimpoint the place where the above proof breaks down if one were to allow formulas containing disjunction. The proof of the lemma would then have to be supplemented with a "case of $v$ ". The 
first half of that case would go through: if $x \in U_{n-1}$, then by the same token as in the case of $\Lambda, \mathcal{U}_{n} k_{x} \beta \vee \gamma$ iff $\mathcal{U}_{n-1} F_{x} \beta \vee \gamma$. The breakdown comes in the latter half: if $x \notin U_{n-1}$, then, if $y_{0}$ and $y_{1}$ are the immediate descendants of $x$, there is, in general, nothing to prevent that

$$
\mathcal{U}_{n} \vDash_{y_{0}} \beta, \quad \mathcal{u}_{n}\left|\neq_{y_{1}} \beta, \quad \mathcal{u}_{n}\right|_{y_{0}} \gamma, \quad \mathcal{u}_{n} \vDash_{y_{1}} \gamma,
$$

whence

$$
\mathcal{U}_{n} \xi_{\nu_{0}} \beta \vee \gamma, \quad \mathcal{U}_{n} \vDash_{y_{1}} \beta \vee \gamma,
$$

\section{A fine hierarchy of partition cardinals}

by

and.

$$
\mathcal{u}_{n} \mid \neq x \beta \vee \gamma \text {. }
$$

\section{References}

[1] D. M. Gabbay and D. H. J. de Jongh, A sequence of decidable finitely axiomatizable intermediate logics with 'the disjunction property, Technical report no. 35 prepared for the U.S. Office of Naval Research, Information Systems Branch, Jerusalem, December 1969.

[2] S. A. Kripke, Semantical analysis of intuitionistio logic I, Formal Systems and Recursive Functions, Amsterdam (1965), pp. 92-130.

Regu par la Rédaction le 28. 4. 1973

Abstract. A modification is made to the definition of a partition cardinal; it is shown that this modified definition gives a finer hierarchy than that used, e.g., in Silver's thesis. Some properties are proved and an application to the constructible universe is given.

1. Introduction. Most of the definitions in use for large cardinals are properties which single out certain cardinals, which from the point of view of all cardinals are few and far between. This in the case, for example, with weakly or strongly compact cardinals, measurable cardinals, or the varions sorts of indescribable cardinals - for all of these, if it seems natural to assume the existence of any of them, it would seem almost equally natural (but at the same time a definite extension) to assume the existence of many of them - perhaps even a proper class of them.

The partition cardinals introduced by Erdös and exploited by Rowbottom, Silver and others, stand out from the other large cardinals in this respect. The vardinal $\varkappa(\alpha)$, for limit ordinal $\alpha$, is defined as the least cardinal $x$ such that $x \rightarrow(\alpha)_{2}^{<\omega}$ (the notation is explained in section 2), and for any $x^{\prime} \geqslant x(\alpha), x^{\prime} \rightarrow(\alpha)_{2}^{<\omega}$ also holds. It is the purpose of this note to show how the definition of $x(\alpha)$ can be modified to yield a notion of " $\alpha$-partition cardinal" which is more analogous to the other large cardinal definitions in that it is a definite extension to assume that there are many snch cardinals, and it is relatively consistent to assume that there is only one (or indeed any fixed ordinal number of them). Of course, the ustat definition gives the hierarchy $\{x(\alpha) \mid \alpha$ a limit ordinal $\}$; our hierarchy will bo a definoment; of this one. An application to the constructible universe is given.

An oarlior parallel is provided by measurable cardinals: at first the question was asked, whother there could be a countably additive, 2-valued mousuro dofined on all subsets of a set, and a cardinal was called measurable if it admitted such a measure. Today we would say that this definition applies to all cardinals $\geqslant$ the first measurable cardinal, and we wll a cardinal $x$ measmrable only if it carries a $x$-additive measure. Our procedure is suggested by this change. 